PROCEEDINGS OF THE

AMERICAN MATHEMATICAL SOCIETY

Volume 29, Number 1, June 1971

\title{
MODULUS SPACE IS SIMPLY-CONNECTED
}

\author{
COLIN MACLACHLAN
}

Abstract. Modulus space of genus $g$ consists of conformal equivalence classes of compact Riemann surfaces of genus $g$. It is the quotient space of the real Teichmuller space of marked Riemann surfaces by the Teichmuller modular group. The result follows using generators and some relations of this group and the fact that it acts discontinuously on Teichmuller space.

1. Let $D$ denote the unit disc $\{z|| z \mid<1\}$ and $\mathscr{L}$ the topological group of conformal homeomorphisms of $D$. A Fuchsian group is a discrete subgroup of $\mathcal{L}$. If $S$ is a compact Riemann surface of genus $g \geqq 2$, then $S$ is conformally equivalent to $D / K_{\theta}$ where $K_{v}$ is a Fuchsian group with presentation:

$$
\begin{array}{cl}
\text { Generators: } & a_{1}, b_{1}, a_{2}, b_{2}, \cdots, a_{\theta}, b_{\theta} \\
\text { Relation: } & \prod_{j=1}^{g} a_{j} b_{j} a_{j}^{-1} b_{j}^{-1}=1 .
\end{array}
$$

Let $K_{g}$ denote a fixed Fuchsian group of the above type and $R\left(K_{g}\right)$ the set of isomorphisms $\phi: K_{g} \rightarrow \mathscr{L} . R\left(K_{g}\right)$ can be topologised by identifying $\phi$ with the point $\left(\phi\left(a_{1}\right), \phi\left(b_{1}\right), \cdots, \phi\left(a_{\theta}\right), \phi\left(b_{\theta}\right)\right)$ of $\mathfrak{2}^{20}$. The group, $\mathfrak{L}^{+}$, of angle-preserving homeomorphisms of $D$, acts on $R\left(K_{g}\right)$ by $(\gamma(\phi))(k)=\gamma \phi(k) \gamma^{-1}$ for $\gamma \in \mathcal{L}^{+}$and all $k \in K_{g}$. The quotient space, $T\left(K_{g}\right)$, is Teichmuller space of genus $g$ and is homeomorphic to $R^{60-6}$. (See [2].)

Let $A\left(K_{g}\right)$ denote the automorphism group of $K_{\theta}$ and $I\left(K_{\theta}\right)$, the inner automorphism group. The quotient $M\left(K_{q}\right)=A\left(K_{g}\right) / I\left(K_{q}\right)$ acts on $T\left(K_{\theta}\right)$ by

$$
\bar{\alpha}[\phi]=[\phi \circ \alpha] \text { where } \bar{\alpha}=\alpha I\left(K_{\vartheta}\right) .
$$

$M\left(K_{\vartheta}\right)$ is a Teichmuller modular group and is a properly discontinuous group of homeomorphisms of $T\left(K_{\theta}\right)$. (See [4].) $T\left(K_{\theta}\right) / M\left(K_{\theta}\right)$ is the modulus space of genus $g$, and is a space of all conformally equivalent compact Riemann surfaces of genus $g$.

2. Lickorish [5] has obtained generators for $M\left(K_{0}\right)$ and Birman [3] some relations between these generators. In proving Theorem 1

Received by the editors August 12, 1970.

A MS 1969 subject classifications. Primary 3045; Secondary 2022, 1451, 5385.

Key words and phrases. Compact Riemann surface, Fuchsian group, Teichmuller space, Teichmuller modular group, generators and relations. 
below we use the methods and notation of [3]. $M\left(K_{\theta}\right)$ is generated by

$$
\left\{h_{u_{i}}, h_{z_{i}}, h_{c}, ; 1 \leqq i \leqq g ; 1 \leqq j \leqq g-1\right\} .
$$

THEOREM 1. $M\left(K_{o}\right)$ can be generated by elements of finite order.

Proof. In [3], it is shown that $\alpha=h_{c_{1}} h_{u_{1}} h_{z_{1}} h_{u_{2}} h_{z_{2}} \cdots h_{u_{g}} h_{z_{g}}$ has finite order $2 g+2$. Using the same methods, one can show that $\beta=h_{u_{1}} h_{z_{1}} h_{u_{2}} h_{z_{2}} \cdots h_{u_{\theta}} h_{z_{g}}$ has finite order $4 g+2$. Let $N$ be the normal subgroup generated by all conjugates of $\alpha$ and $\beta$ so that $N$ is generated by elements of finite order. Then $M\left(K_{g}\right) / N$ will be generated by

$$
\left\{\bar{h}_{u_{i}}, \bar{h}_{z_{i}}, \bar{h}_{c_{j}} ; 1 \leqq i \leqq g, 1 \leqq j \leqq g-1\right\}
$$

which satisfy all the relations of $M\left(K_{g}\right)$ together with the additional relations $\bar{\alpha}=1, \bar{\beta}=1$. But these imply that $\bar{h}_{c_{1}}=1$. Repeated application of relations (7), (8) and (9) of [3], then gives $\bar{h}_{c_{j}}=\bar{h}_{u_{i}}=\bar{h}_{z_{i}}=1$ for all $i, j$. Thus $M\left(K_{g}\right)=N$.

TheOREM 2. Modulus space is simply-connected.

Proof. It is proved in [4] that each element of finite order in $M\left(K_{g}\right)$ has a fixed point in $T\left(K_{g}\right)$, so that, by Theorem $1, M\left(K_{g}\right)$ is generated by elements which have fixed points. Also $M\left(K_{g}\right)$ is a properly discontinuous group of homeomorphisms of a space homeomorphic to $R^{6 g-6}$. Furthermore, the stabiliser of a point $[\phi]$ of $T\left(K_{g}\right)$ is isomorphic to the group of conformal self-homeomorphisms of the compact Riemann surface $D / \phi\left(K_{o}\right)$ and hence is finite. Thus, applying a result of Armstrong [1] we have that $T\left(K_{g}\right) / M\left(K_{o}\right)$ has trivial fundamental group.

\section{REFERENCES}

1. M. A. Armstrong, The fundamental group of the orbit space of a discontinuous group, Proc. Cambridge Philos. Soc. 64 (1968), 299-301. MR 36 \#4540.

2. L. Bers, Quasiconformal mappings and Teichmuller's theorem, Analytic Functions, Princeton Univ. Press, Princeton, N. J., 1960, pp. 89-119. MR 22 \#5716.

3. J. S. Birman, Automorphisms of the fundamental group of a closed orientable 2-manifold, Proc. Amer. Math. Soc. 21 (1969), 351-354. MR 39 \#950.

4. S. Kravetz, On the geometry of Teichmiiller spaces and the structure of their modular groups, Ann. Acad. Sci. Fenn. Ser. AI No. 278 (1959). MR 26 \#6402.

5. W. B. R. Lickorish, A finite set of generators for the homeotopy group of a 2-manifold, Proc. Cambridge Philos. Soc. 60 (1964), 769-778. MR 30 \#1500.

University of Aberdeen, Aberdeen, Scotland 